\title{
Static Transmission Expansion Planning using Heuristic and Metaheuristic Techniques
}

\author{
Phillipe Vilaça Gomes \\ INESC TEC and FEUP/DEEC \\ Dept. Eng. Eletrotécnica e Computadores da \\ Faculdade de Engenharia da Universidade do Porto \\ Porto, Portugal \\ phillipe.gomes@fe.up.pt
}

\author{
João Tomé Saraiva \\ INESC TEC and FEUP/DEEC \\ Dept. Eng. Eletrotécnica e Computadores da \\ Faculdade de Engenharia da Universidade do Porto \\ Porto, Portugal \\ jsaraiva@fe.up.pt
}

\begin{abstract}
This paper describes a hybrid tool to perform Static Transmission Expansion Planning, STEP, studies and its application to the Garver6-Bus academic system and to the Southern Brazilian Transmission equivalent real system. The developed STEP tool integrates two phases as follows. The first one uses Constructive Heuristic Algorithms (CHA) to reduce the search space, and the second uses Particle Swarm Optimization (PSO) to identify the final solution. This hybridization between CHAs and PSO proved to be very effective and shows good performance to reduce the size of the STEP search space and to identify good quality solutions. These are relevant issues given the combinatorial nature of investment problems leading to the explosion of the number of alternative plans, one of the greatest difficulties faced in this planning problem.
\end{abstract}

Index Terms - Constructive Heuristic Algorithms, Particle Swarm Optimization, Reduction of the Search Space Size, Transmission Expansion Planning.

\section{INTRODUCTION}

The purpose of STEP, Static Transmission Expansion Planning problems, is to determine how the transmission capacity of a network should be enlarged, satisfying the increasing demand, i.e, from a pre-existing list of candidate circuits to be built, identify those that will be constructed to minimize the operation cost of the system while supplying the forecasted demand along a planning horizon [1]. The increasing demand implies modifying the system over time so that the load is properly supplied. There are usually different alternatives to do this that include not only building new transmission lines but also installing new generation facilities closer to the demand centers. In whatever way, it is frequently not economically feasible or even possible to build generating units near the demand centers. On the other hand, transmission expansion can also enable the optimal dispatch of power plants, because having a stronger transmission system provides more flexibility to dispatch generation apart from improving the reliability of the system and decreasing the likelihood of having congested branches.
Solving the STEP optimization problem is an arduous task since it has some special features that increase its complexity:

- The search space is non-convex so that several solution algorithms may converge to local optima;

- In some cases there are isolated smaller systems;

- The problem typically has an integer nature leading to the combinatorial explosion of investment alternative plans that can be devised. This requires a high computational effort to identify good quality plans.

These characteristics correspond to the main difficulties in developing high-performance tools in terms of speed, efficiency and robustness to solve the STEP problem [2]. The literature of this area includes a variety of models and tools to solve the STEP and to address the difficulties mentioned above. These models and tools can be organized as follows:

- Classical Optimization Algorithms

They use decomposition techniques and generally find global optimal solutions for a relaxed version of the original integer problem in which integer investment alternatives are substituted by continuous variables. However, they usually require a large computational effort and therefore they display difficulties to address the expansion of medium and large systems as most of real transmission systems are. In some cases they can also show convergence problems as detailed in [3], [4];

- Constructive Heuristics Algorithms (CHAs)

These approaches correspond to simplified procedures that are suitable to identify feasible solutions for complex problems using efficient and easily applied algorithms. They have little computational effort, but they rarely find the optimal global solution, especially if one is addressing real transmission networks [5] , [6];

- Metaheuristics

They are heuristic techniques that are enhanced with particular search procedures in most cases inspired in natural mechanisms. They are especially suited to solve complex and combinatorial problems usually identifying optimal or suboptimal solutions even for large systems. 
However, they are typically associated to large computational efforts [7].

Given the shortcomings of the above approaches, this paper details the application of an hybridizing methodology to solve the STEP problem. This hybridization results in an algorithm that incorporates two phases. The first phase is termed The reduction of search space size and it uses two $\mathrm{CHAs}$ with different modeling of the problem: the Garver CHA and the Minimum Effort CHA. In the second phase, termed as The refinement of the solution, we used Particle Swarm Optimization (PSO) to search for the global optimum solution in the reduced search space, that is, in the output of the first phase. This process was designed to overcome the problem associated to large computational effort that is typical to metaheuristics. Accordingly, we promote a reduction of the search space and only afterwards PSO is used to search for the most adequate solution in the reduced solution space.

Regarding the structure of the paper, following the introduction already presented, Section II refers to the mathematical model for TEP problem, Section III details the CHA theory, Section IV provides a brief presentation of the PSO approach and the hybridization of these two approaches in the context of the STEP problem. Section V presents the results of PSO and hybrid tool applied to the Garver-6-bus and to the Equivalent System Southern Brazil test systems as well as the considerations adopted for these simulations. Finally, Section VI discusses the results of the simulations, as well as the behavior of the proposed tool and presents the main conclusions of this paper.

\section{MATHEMATICAL MODELING OF THE STEP PROBLEM}

The complete mathematical model for the STEP problem uses the AC model for the operation of the power system as described in [8]. However, this model has many obstacles to its widespread use. One possible simplification results from only addressing active power flows in the STEP problem leaving the reactive planning issues to a subsequent phase. Another problem is related with the presence of sets of islanded buses in the starting configuration. Accordingly, most of the STEP models are based on relaxed versions of the full AC version. Such models are the Transportation Model, the DC Model and Hybrid Models. The developed approach incorporates two CHAs in phase 1, the Garver and the Minimum Effort CHA. These two CHAs use the Transportation Model and the DC Model, respectively. The formulation based on the DC model is detailed below [9] .

$$
\begin{aligned}
& \operatorname{minimize} v=\sum_{(i, j)} c_{i j} n_{i j}+\alpha \cdot \sum r_{k} \\
& \text { subject to } \quad S . f+g+r=d \\
& f_{i j}-\left(\gamma_{i j}^{0}+x_{i j}\right) \cdot\left(\theta_{i}-\theta_{j}\right)=0 \\
&\left|f_{i j}\right| \leq\left(x_{i j}+\gamma_{i j}^{0}\right) \cdot \bar{\phi}_{i j} \\
& 0 \leq g_{i} \leq \bar{g}_{i} \\
& 0 \leq \eta_{i j} \leq \bar{\eta}_{i j}
\end{aligned}
$$

Regarding the nature of the variables, $\eta_{i j}$ integer, $x_{i j}$ discrete, and $f_{i j}$ and $\theta_{i}$ are unrestricted. On the other hand, $c_{i j}$ is the cost of a circuit $\mathrm{ij}, \eta_{i j}$ is the number of circuits to be built from i to $\mathrm{j}, \alpha$ is a factor that penalizes load shedding in the bus k modeled by $r_{k}, S$ is the transposed incidence nodebranch matrix of the power system, $f$ is the vector of the branch flows, $g$ is the generation vector, $d$ is the demand vector, $\gamma_{i j}^{0}$ is the equivalent susceptance of circuit $i-j$ in the base topology, $x_{i j}$ is the susceptance of the added $\mathrm{i}-\mathrm{j}$ circuit, $\theta$ is the voltage angle, and finally $\phi_{i j}=\overline{f_{i j}} / \gamma_{i j}$.

The Transportation Model differs from the DC based Model because it does not use the second Kirchhoff law, i.e, the equality constraints (3). These models are usually known as the associated problems to the STEP problem.

The size of the search space $(S)$ of STEP problem can be calculated taking into account the maximum number of circuits that can be built into candidates routes $(n)$ and the number of existing candidate routes $(p)$, as shown in (7).

$$
S=(n+1)^{p}
$$

\section{CONSTRUCTIVE HEURISTIC ALgORITHMS}

These algorithms analyze the search space using a number of sensitivity criteria interpreted as quantifiers used to evaluate how the system performs if some lines are added to the original system. Therefore, in each step a circuit is selected and tested and it is eventually selected to remain in the reduced search space. The sensitivity criteria correspond to parameters that measure how the objective function of the problem changes if a circuit is added. It is then clear that they should be able to identify the most attractive circuits to be added, and correspond to indicators having a local character. However, they do not ensure that the CHA reduces the search space in a way that the global optimum is included in it. The generic CHA is shown in Fig. 1 bellow. In the developed approach, this tool was used in an auxiliary way, i.e, its output is used just to reduce the search space.

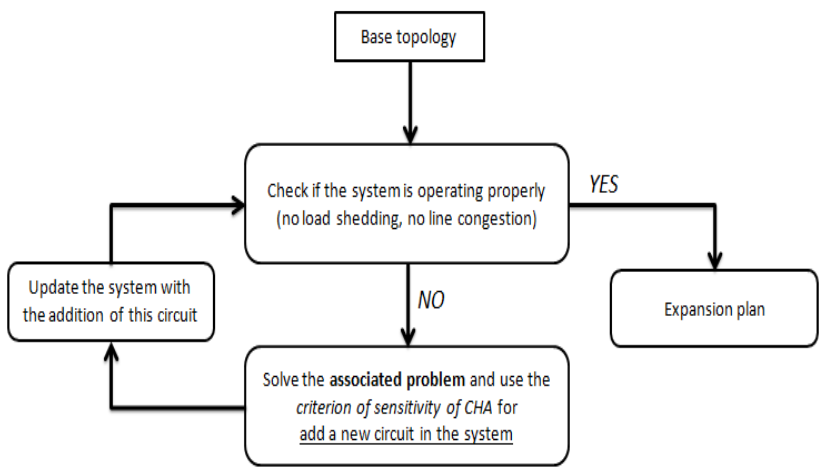

Figure 1. Illustration of a generic $\mathrm{CHA}$

The Garver CHA uses the Transportation Model to solve the STEP problem and uses as criterion the sensitivity of the flows associated to the new circuits that can be added to the system. This sensitivity is modeled by (8) as defined in [3]. 
The search reduction promoted by this CHA may not be the most interesting (compared to the one obtained with Minimum Effort approach) because it uses the Transportation Model. However, the literature indicates that it outputs acceptable results for complex and islanded systems.

$$
C S^{G}=n_{i j} \cdot \overline{f_{i j}}
$$

The Minimum Effort CHA uses the DC Model as an associated problem and its sensitivity criterion is given by (9) as detailed in [5]. This approach is more refined than the previous one and therefore it usually provides better results, but it presents difficulties in dealing with islanded systems. This can be addressed by creating a fictitious network overlaid with the existing one connecting the entire system. However, even using this artificial mechanism, the Minimum Effort CHA can display convergence problems in some cases.

$$
C S^{M E}=\frac{1}{2} \cdot\left(\theta_{i}-\theta_{j}\right)^{2} \cdot \gamma_{i j}
$$

As the Garver CHA is a relaxed technique, it displays fewer convergence problems to get a reduction of the search space. However, the Minimum Effort CHA models real problems in a closer way and therefore it can provide better answers, in most cases. So, it seems advantageous to combine these two techniques in order to build a reduced search space profiting from the characteristics of both approaches. Accordingly, the output of the first phase of the developed algorithm to solve the STEP problem (reduction of the search space size) corresponds to the union of the search spaces that are obtained using these two CHAs.

\section{PARTICLE SWARM OPTIMIZATION - PSO}

PSO is a swarm intelligence technique and a stochastic optimization algorithm based on social simulation models. The development of PSO was based on concepts which govern socially organized populations in nature, such as bird flocks, fish schools and animal herds [10]. This technique basically employs a set of points (population) that moves in the search space. The best position reached by each point is maintained, and then communicated to all particles of the swarm. Each of these particles is characterized by a value that measures the suitability of the particle as a solution to the problem. Each particle evolves along the solution algorithm using a velocity vector that defines the direction of its movement. The swarm is successful over time because the position of each particle is updated, taking into account the best position of the particle in the past generations and the best position of all particles in the swarm. The velocity of each particle is given by (10) and the position is obtained by (11).

$$
\begin{gathered}
V_{i}^{k+1}=w v_{i}^{k}+c_{1} \cdot \text { rand }_{1} \cdot\left(\text { pbest }_{i}-s_{i}^{k}\right)+c_{2} \cdot \text { rand }_{2} \cdot\left(\text { gbest }_{i}-s_{i}^{k}\right) \\
s_{i}^{k+1}=s_{i}^{k}+v_{i}^{k+1}
\end{gathered}
$$

In this expression $V_{i}^{k}$ is the velocity of particle $\mathrm{i}$ at iteration $\mathrm{k}, \mathrm{w}$ is a weighting function, $c_{1}$ and $c_{2}$ are weighting coefficients, rand is a random number in $[0,1]$, pbest is the best particle in previous generations, $S_{i}^{k}$ is the position of particle $\mathrm{i}$ at iteration $\mathrm{k}$ and gbest is the best particle in the entire swarm. This movement rule is illustrated in Fig. 2.

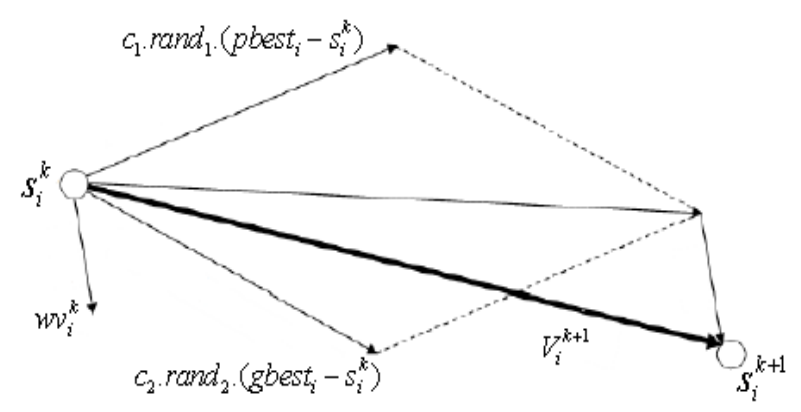

Figure 2. Movement rule of the PSO algorithm.

The stopping criterion is usually given by a function that establishing a maximum or a minimum range for the evolution of PSO parameters or a maximum number of iterations. Regarding expression (10) the first term $w v_{i}^{k}$ refers to the moment of inertia of the particle. The second term $c_{1}$.rand r $_{1}\left(\right.$ pbest $\left._{-} s_{i}^{k}\right)$ refers to the "cognitive" part, which represents the individual knowledge of the particle acquired over the search process. The third term $c_{2}$.rand $2 .\left(\right.$ gbest $\left._{i}-s_{i}^{k}\right)$ refers to the "social" part, which is the collaboration between the particles, i.e, the collective knowledge gained from the swarm throughout the search process. The second and third terms are weighted by two constants $\left(c_{1}\right.$ and $\left.c_{2}\right)$ that represent the weighting of the individual and collective components respectively and influence each particle towards the new solution, while the first term is weighted by a function $(w)$, called inertial weighting function, that induces the particle to move in a direction based on the move of the previous iteration. Larger values of the weighting function facilitate a global search while smaller values tend to represent a local one. Results provided in the literature mention that it is better to adjust the weighting function in a larger value at the beginning of the search process, promoting a more comprehensive search, and gradually, throughout the process, reduce it to refine the search [11]. Therefore, in the developed approach we used for $w$ values of $0.9\left(w_{\max }\right)$ in the beginning of the process and of $0.4\left(w_{\min }\right)$ in the end of the process in accordance with (12). In this expression iteration $_{\max }$ is the maximum number of iterations allowed in the process and iteration represents the current iteration.

$$
w=w_{\max }-\frac{w_{\max }-w_{\min }}{\text { iteration }_{\max }} \text {.iteration }
$$

The PSO tool was used in this approach as an auxiliary tool with the purpose of refining the solution provided by the CHAs. Fig. 3 illustrates the hybrid tool that was developed to solve the STEP problem. It includes the two mentioned CHA algorithms to promote a reduction of the search space and then PSO is used over this reduced search space to identify the most adequate expansion plan. 


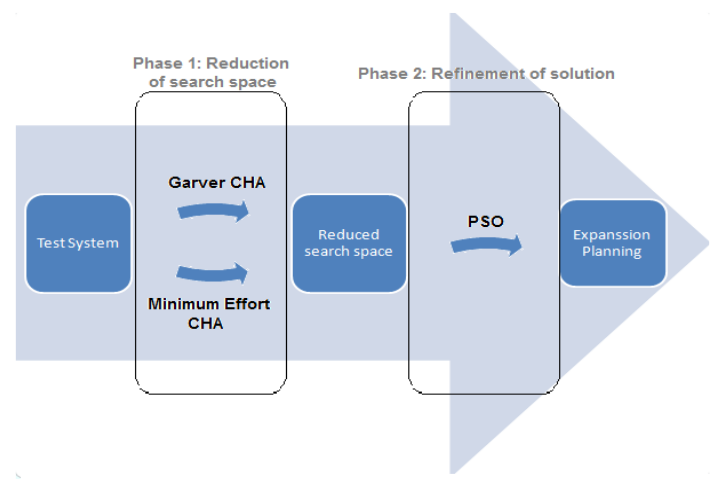

Figure 3. Flow of the tools used in the developed approach.

\section{RESULTS}

This section presents the results obtained using the hybrid proposed tool applied to the Garver 6-bus and to the Southern Brazilian equivalent 46-bus networks. For comparison purposes we are also presenting the results that are obtained by just using the classical PSO algorithm without the previous applying the described CHA's techniques. It is also important to indicate that all test were conducted admitting that the generation could be rescheduled and also without this possibility. Not considering rescheduling means that the generation pattern is pre-determined in order to supply the demand while if rescheduling is considering then generation outputs can be change in order to minimize the overall cost. It is then clear that admitting rescheduling gives the problem extra flexibility and so the expansion cost will be smaller when compared with the one without rescheduling. The algorithm was implemented in MATLAB, running on an Intel i5, $2.53 \mathrm{GHz}, 4 \mathrm{~GB}$ RAM, hardware platform. The tests were performed considering power generation with and without reschedule generation.

\section{A. Garver 6-Bus System}

This system consists of 6 bars, 6 existing circuits at the base topology, 15 candidate circuits for expansion admitting that it is allowed to build up to 4 circuits in each path, and forecasted demand for the planning horizon of $760 \mathrm{MW}$. Therefore, the size of the search space of the STEP problem is $5^{15}$. The system data and topology can be obtained in [1].

\section{A.1. Case 1 - With Rescheduled Generation}

To simulate the Garver 6-bus system with reschedule generation we considered the following parameters: (i) $1 \mathrm{MW}$ tolerance for the total load shedding; (ii) swarm composed of 100 particles; (iii) stopping criterion of 100 iterations or 20 iterations with the same best global result (gbest); (iv) $c_{1}=c_{2}=2$ and (v) $\alpha=100$.

Using these parameters, the proposed tool was applied. In the first phase, reduction of the search space size, 3 circuits $\left(n_{2-6}, n_{3-5}, n_{4-6}\right)$ were selected in just 0.4 seconds, which has reduced the search space from $5^{15}$ to $5^{3}$, i.e, a reduction larger than $99 \%$. In the second phase, refinement of the solution, the PSO was applied over the reduced search space and the best known solution was obtained with additions in $n_{3-5}=1$ and $n_{4-6}=3$. The cost of this expansion plan is US\$ 110 . The process converged to the solution in 23 iterations solving 2300 optimization problems (associated problems to the STEP problem) in 30.3 seconds, which results in a total computation time of approximately 31 seconds. Fig. 4 shows the evolution of the expansion cost of the gbest particle, that is the cost of the particle that in each iteration of the PSO algorithm has the lowest cost.

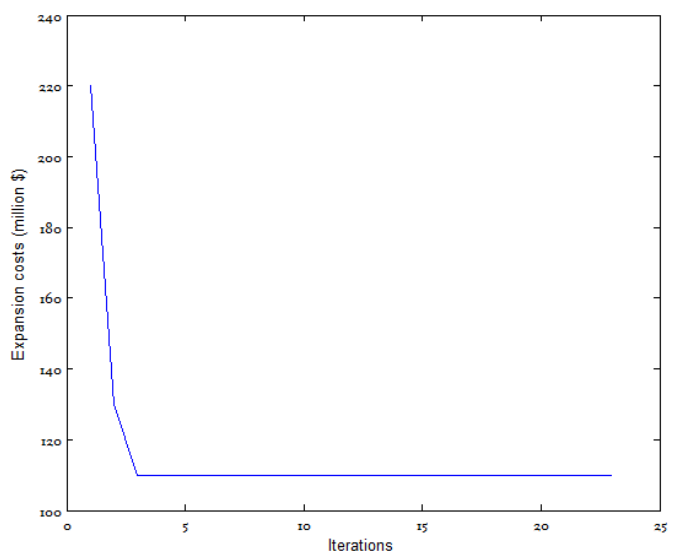

Figure 4. Evolution of the expansion cost along the PSO algorithm Garver 6-bus system - Case 1.

In order to evaluate the performance of the proposed hybrid tool, we used the PSO algorithm without considering the CHA's techniques. In this case, considering the same parameters indicated previously, the PSO provided the same best solution after running 26 iterations solving 2600 optimization problems in approximately 55 seconds. Table 1 shows the main results that were obtained in these tests.

TABLE I. GARVER 6-BUS SYSTEM WITH RESCHEDULE GENERATION

\begin{tabular}{|c|c|c|c|c|}
\hline \multirow{2}{*}{ Tool } & \multicolumn{4}{|c|}{ Results obtained } \\
\cline { 2 - 5 } & $\begin{array}{c}\text { Reduction of the } \\
\text { search space in } \\
\text { phase 1 }\end{array}$ & $\begin{array}{c}\text { Expansion } \\
\text { costs (US\$) }\end{array}$ & Iterations & Time (s) \\
\hline CHA+PSO & $99 \%$ & 110 & 23 & 31 \\
\hline PSO & --- & 110 & 26 & 55 \\
\hline
\end{tabular}

\section{A.2. Case 2 - Without Rescheduled Generation}

In this simulation we considered the same parameters as in Case 1 . The proposed tool was applied and in phase 1 it has selected 4 circuits $\left(n_{2-6}, n_{3-5}, n_{4-6}, n_{5-6}\right)$ in just 0.7 seconds, which has reduced the search space from $5^{15}$ to $5^{4}$, i.e, again a reduction larger than $99 \%$. Then, the PSO was applied in this reduced search space and one of the best known solution was obtained corresponding to additions in $n_{2-6}=3, n_{3-5}=1$ and $n_{4-6}=3$, with an expansion cost of US\$200. The process converged to the solution in 31 iterations solving 3100 optimization problems in 51 seconds, therefore resulting in a total computation time of approximately 52 seconds. Fig. 5 shows the evolution of the cost that was obtained.

Once again, the classical PSO was also applied considering the same previous parameters. In this case the 
same best solution was obtained with the difference that the process converged to the solution in 48 iterations solving 4800 optimization problems in approximately 215 seconds. The Table 2 shows the main results obtained for the Garver 6-Bus System without reschedule generation.

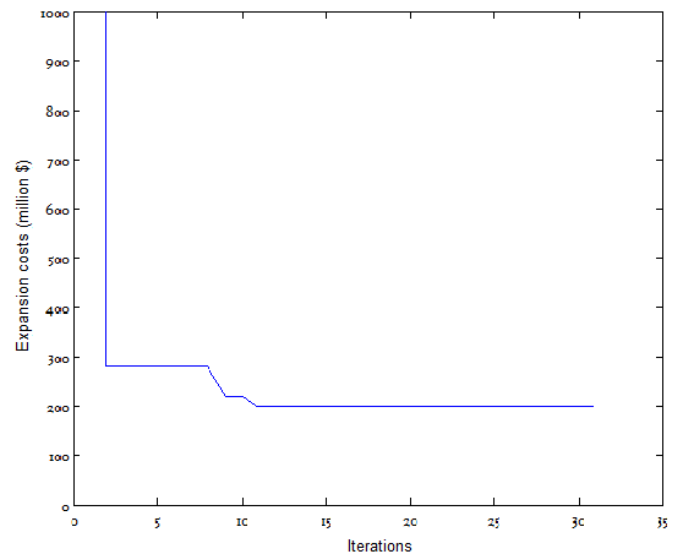

Figure 5. Evolution of the expansion cost along the PSO algorithm Garver 6-bus system - Case 2.

TABLE II. GARVER 6-BUS WITHOUT RESCHEDULE GENERATION.

\begin{tabular}{|c|c|c|c|c|}
\hline \multirow{2}{*}{ Tool } & \multicolumn{4}{|c|}{ Results obtained } \\
\cline { 2 - 5 } & $\begin{array}{c}\text { Reduction of the } \\
\text { search space in } \\
\text { phase 1 }\end{array}$ & $\begin{array}{c}\text { Expansion } \\
\text { costs (US\$) }\end{array}$ & Iterations & Time (s) \\
\hline CHA+PSO & $99 \%$ & 200 & 31 & 51 \\
\hline PSO & --- & 200 & 48 & 215 \\
\hline
\end{tabular}

\section{B. Southern Brazilian Equivalent 46-Bus System}

This is an equivalent system and of the southern part of the Brazilian interconnected system. It consists of 46 buses and 79 candidate paths for expansion and we admitted that it is allowed to build up to 2 circuits in each path. The total demand for this system is $6880 \mathrm{MW}$. The size of the search space of this system is $3^{79}$. The system data and topology can be obtained from [12].

\section{B.1. Case 1 - With Rescheduled Generation}

In this simulation we considered the following parameters: (i) $1 \mathrm{MW}$ tolerance for the total load shedding; (ii) swarm composed by 100 particles; (iii) stopping criterion of 100 iterations or 20 iterations with the same best global result (gbest); (iv) $c_{1}=c_{2}=2$ and (v) $\alpha=100$.

Using these parameters, the proposed tool was applied and in the first phase 22 circuits were selected $\left(n_{2-3}, n_{3-46}, n_{5-6}, n_{6-46}, n_{12-14}, n_{13-20}, n_{16-32}, n_{17-19}, n_{19-21}, n_{19-25}, n_{20-21}\right.$, $\left.n_{20-23}, n_{21-25}, n_{24-25}, n_{28-31}, n_{28-43}, n_{31-32}, n_{31-41}, n_{32-43}, n_{40-41}, n_{40-45}, n_{42-43}\right)$ in 4.88 seconds. This provided a reduction of the search space from $3^{79}$ to $3^{22}$, i.e, a reduction larger than $99 \%$. Thus, in the second phase the PSO was applied over the reduced search space and the best known solution for Southern Brazilian equivalent system with reschedule generation was obtained, with the following additions $\eta_{5-6}=2, \quad \eta_{6-46}=1, \quad \eta_{13-20}=1$, $\eta_{10-21}=2, \eta_{20-23}=1$ and $\eta_{42-43}=1$. The cost of this expansion plan is US\$ 70,289.000. The process converged to this solution in 45 iterations solving 4500 optimization problems in 984 seconds, which results in a total computation time of approximately 989 seconds. Fig 6 shows the evolution of expansion cost along the PSO algorithm.

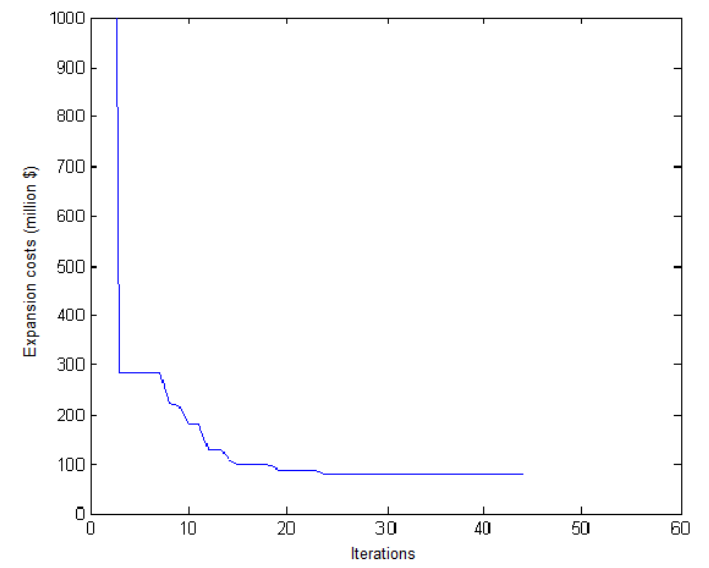

Figure 6. Evolution of the expansion cost along the PSO algorithm Southern Brazilian - Case 1 .

Once again, in order to evaluate the performance of the proposed hybrid tool, the PSO was applied alone considering the same previous parameters. The same solution was obtained with an expansion costs US\$ 70,289.000. In this case, the process converged to this solution in 59 iterations solving 5900 optimization problems in 1392 seconds, that is, with more computational effort than the proposed tool. Table III shows the main results obtained in these two simulations.

TABLE III. SOUTHERN BRAZILIAN WITH RESCHEDULE GENERATION WITH RESCHEDULED GENERATION.

\begin{tabular}{|c|c|c|c|c|}
\hline \multirow{2}{*}{ Tool } & \multicolumn{4}{|c|}{ Results obtained } \\
\cline { 2 - 5 } & $\begin{array}{c}\text { Reduction of the } \\
\text { search space in } \\
\text { phase 1 }\end{array}$ & $\begin{array}{c}\text { Expansion } \\
\text { costs (US\$) }\end{array}$ & Iterations & Time (s) \\
\hline CHA+PSO & $99 \%$ & $70,289.000$ & 45 & 989 \\
\hline PSO & --- & $70,289.000$ & 59 & 1392 \\
\hline
\end{tabular}

\section{B.2. Case 2 - Without Rescheduled Generation}

The Southern Brazil Equivalent system without reschedule generation was also simulated using the same parameters that were indicated for the previous case. In this simulation we admitted that up to 3 circuits can be built in each candidate path. Therefore, the size of the search space of this system is $4^{79}$. The proposed hybrid tool was applied and in phase 1 has selected 27 circuits in 16 seconds, namely: $n_{2-3}, n_{3-46}, n_{5-6}, n_{6-46}, n_{9-10}, n_{10-46}, n_{12-14}, n_{16-28}, n_{16-32}, n_{17-19}$, $n_{19-21}, n_{19-25}, n_{20-21}, n_{21-25}, n_{24-25}, n_{25-32}, n_{26-29}, n_{28-30}, n_{28-31}$, $n_{28-43}, n_{29-30}, n_{31-32}, n_{31-41}, n_{32-43}, n_{40-41}, n_{40-45}, n_{42-43}$. This reduced the search space from $4^{79}$ to $4^{27}$, i.e, again a reduction larger than $99 \%$. As in the previous cases, the PSO was then applied over this reduced search space and the best known solution was obtained corresponding to additions in $\eta_{5-6}=2, \quad \eta_{6-46}=1, \quad \eta_{19-25}=1, \quad \eta_{20-21}=1, \quad \eta_{24-25}=2$, $\eta_{26-29}=3, \quad \eta_{28-30}=1, \quad \eta_{29-30}=2, \quad \eta_{31-32}=1 \quad$ and $\quad \eta_{42-43}=2$ 
with an expansion cost of US\$ $154,420.000$. The process converged to the solution in 64 iterations solving 6400 optimization problems in 1540 seconds, therefore, resulting in a total computation time of approximately 1556 seconds. Fig. 7 shows the evolution of the expansion cost.

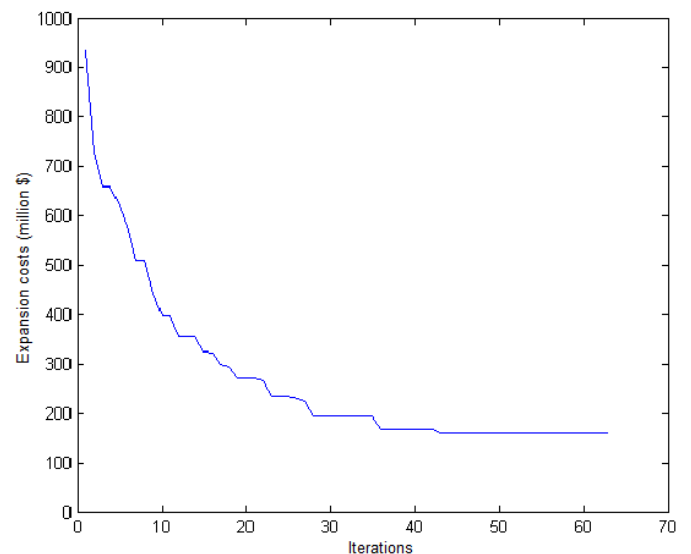

Figure 7. Evolution of the expansion cost along the PSO algorithm Southern Brazilian - Case 2.

Finally, the PSO alone was applied considering the same previous parameters and a new solution was obtained with the addition of 16 circuits: $\eta_{5-6}=2$, $\eta_{6-46}=1, \eta_{16-28}=1, \quad \eta_{18-20}=1, \quad \eta_{20-21}=2, \quad \eta_{20-23}=2$, $\eta_{31-32}=1, \eta_{32-41}=1, \eta_{40-41}=3$ and $\eta_{40-42}=2$. The cost of this expansion plan is US\$ $180,430.000$. The process converged to this solution in 81 iterations solving 8100 optimization problems in 1890 seconds. Finally, Table IV shows the main results obtained for the Southern Brazil Equivalent system without reschedule generation.

TABLE IV. SOUTHERN BRAZILIAN WITHOUT RESCHEDULE GENERATION

\begin{tabular}{|c|c|c|c|c|}
\hline \multirow{2}{*}{ Tool } & \multicolumn{4}{|c|}{ Results obtained } \\
\cline { 2 - 5 } & $\begin{array}{c}\text { Reduction of the } \\
\text { search space in } \\
\text { phase 1 }\end{array}$ & $\begin{array}{c}\text { Expansion } \\
\text { costs (US\$) }\end{array}$ & Iterations & Time (s) \\
\hline CHA+PSO & $99 \%$ & $154,420.000$ & 64 & 1556 \\
\hline PSO & --- & $180,430.000$ & 81 & 1890 \\
\hline \multicolumn{5}{|c|}{ CONCLUSIONS } \\
\hline
\end{tabular}

This paper describes a hybrid tool to solve the STEP problem. This tool is structured in two phases as follows. The first one is performed through two heuristics, Garver CHA and Minimum Effort CHA in order to reduce the set of candidate routes, i.e, to reduce efficiently the search space of the STEP problem. In the second phase we used PSO to go over the selected routes from first phase in order to refine the solution resulting in an expansion plan. This tool was applied in two test systems, Garver 6-bus and Southern Brazil 46-bus. The Garver system, although being a small system, is widely used in the literature for comparison purposes. The second tested network is based on the Southern Brazilian transmission system and given its size it allows taking more meaningful conclusions on the performance of the developed approach. In all tested situations, it was possible to reduce the search space more than $99 \%$ after completing the first phase. At the end of the second phase, the final expansion plan provided by the combined use of the two CHA's and the PSO is the same or is better than the one that is obtained by just using the PSO as indicated in Tables I to IV.

These results confirm that the hybridization between CHAs and PSO used to reduce the search space and to identify the final solution is a powerful tool because it has the ability to find adequate expansion plans in an efficient way when compared with other techniques. Therefore, future work will be developed in this area namely to continue testing other hybridization combinations and also to pass from a single period static analysis to a multiperiod formulation.

\section{ACKNOWLEDGMENT}

The first author thanks CAPES Foundation, Ministry of Education of Brazil, for financing this research.

This work is financed by the FCT - Fundação para a Ciência e a Tecnologia (Portuguese Foundation for Science and Technology) within project UID/EEA/50014/2013.

\section{REFERENCES}

[1] G. Latorre, R. D. Cruz, J. M. Areiza, A. Villegas, "Classification of publications and models on transmission expansion planning", IEEE Transactions on Power Systems, vol. 18, no. 2, pp. 938- 946, May. 2003.

[2] I. M. Mendonça, C. S. Ivo, A. L. M. Marcato, B. H. Dias "Transmission expansion optimization via constructive heuristic technique", 2013 IEEE Grenoble PowerTech, POWERTECH, pp. 1-5, June 2013.

[3] L. L. Garver, "Transmission Network Estimation Using Linear Programming," IEEE Trans. Power Apparatus and Systems, vol. PAS89, n 7, p. 1688-1697, Sep. 1970.

[4] I. G. Sánchez, R. Romero, J. R. S. Mantovani and M. J. RIDER. "Transmission - Expansion Planning Using the DC Model and Nonlinear - Programming Technique". IEE Proceedings of Generation, Transmission and Distribution, vol. 152, no. 6, pp. 763-769, Nov 2005.

[5] M. Rider, A. V. Garcia, R. Romero, "Transmission System Expansion Planning by a Branch-and-Bound Algorithm", IET Generation, Transmission \& Distribution, vol. 2, no. 1, pp. 90-99, January 2008.

[6] Z. Michalewicz, D. Fogel, How to solve it: Modern heuristic, vol. I. New York: Springer, 2004.

[7] M. C. Rocha, J. T. Saraiva, "Multiyear Transmission Expansion Planning Using Discrete Evolutionary Particle Swarm Optimization", Energy Market (EEM), 2011 - 8th International Conference on the European, pp. 802 - 807, May 2011.

[8] A. M. L. Silva, L. S. Rezende, L. A. F. Manso, L. C. Resende "Reliability worth applied to transmission expansion planning based on ant colony system", International Journal of Electrical Power \& Energy Systems, vol. 32, no. 10, pp. 1077-1084, Jul. 2010.

[9] C. T. Miasaki, E. M. C. Franco, R. A. Romero " Transmission network expansion planning considering phase-shifter transformers ", Journal of Electrical and Computer Engineering, vol. 2012, no. 6, Jan. 2012.

[10] J. Kennedy, R. Eberhart, Swarm Intelligence, USA, Academic Press, 2001.

[11] I. Miranda de Mendonça, I. Chaves Silva Junior, A. L. M. Marcato, "Static planning of the expansion of electrical energy transmission systems using particle swarm optimization", International Journal of Electrical Power \& Energy Systems, vol. 60, pp. 234-44, Sept. 2014.

[12] A. Monticelli, A. Santos, M. V. Pereira, S. H. Cunha, B. J. Parker, J. C. Praça, "Interactive transmission network planning using a least-effort criterion", IEEE Transactions on Power Apparatus and Systems, vol. PAS-101, no 10, pp. 3919-3925, Feb. 2007. 